UPRF 96-488

IFUM-565-FT

November 1996

\title{
Infrared renormalons and finite volume [
}

\author{
F. Di Renzo ${ }^{a}$, G. Marchesini ${ }^{b}$ and E. Onofri ${ }^{c}$ \\ ${ }^{a}$ Department of Mathematical Sciences, \\ University of Liverpool, United Kingdom \\ ${ }^{b}$ Dipartimento di Fisica, Università di Milano \\ and INFN, Sezione di Milano, Italy \\ ${ }^{c}$ Dipartimento di Fisica, Università di Parma \\ and INFN, Gruppo Collegato di Parma, Italy
}

\begin{abstract}
We analyze the perturbative expansion of a condensate in the $O(N)$ non-linear sigma model for large $N$ on a two dimensional finite lattice. On an infinite volume this expansion is affected by an infrared renormalon. We extrapolate this analysis to the case of the gluon condensate of Yang-Mills theory and argue that infrared renormalons can be detected by performing perturbative studies even on relatively small lattices.
\end{abstract}

*Research supported in part by MURST, Italy and by EC Programme "Human Capital and Mobility", contract CHRX-CT92-0051 and contract CHRX-CT93-0357. 


\section{Introduction}

In $S U(3)$ Yang-Mills theory on the lattice, the coefficients of the perturbative expansion of the simplest observables, the elementary Wilson plaquettes, have been computed to large order [1, 2] and they show evidence of factorial growth. These perturbative calculations are needed not only to clarify the presence and relevance of infrared (IR) renormalons [3] but also to extract the values of physical quantities, such as heavy quark decay constants [4] from the results of Monte Carlo simulations.

The perturbative coefficients of the elementary Wilson plaquette, an observable corresponding to the $S U(3)$ gluon condensate, are known analytically [1] up to the third order in $\alpha_{S}$, the weakcoupling parameter at the ultraviolet lattice scale, related to the usual lattice constant $\beta$ by $4 \pi \alpha_{S}=$ $6 / \beta$. At larger orders these coefficients have been obtained [2] by a numerical method based on the stochastic formulation of the theory à la Parisi-Wu [5]. One considers the weak coupling expansion of the Langevin equations and solves numerically the truncated set of equations corresponding to a given perturbative order. The coefficients have been computed numerically up to the order $\alpha_{S}^{8}$ on a $8^{4}$ lattice and up to the order $\alpha_{S}^{6}$ on a $12^{4}$ lattice. In the sequel we shall always consider symmetric square $(\mathrm{d}=2)$ or hypercubic $(\mathrm{d}=4)$ lattices with $M$ lattice points in each direction.

Up to the sixth order the differences between the coefficients for $M=8$ and $M=12$ are found to be contained within the statistical errors, at present smaller than $10 \%$.

An important question in lattice calculation is to what extent the finite volume affects the results. In particular, if the perturbative part of an observable is affected by an IR renormalon, the factorial behaviour of its coefficients is tamed by the finite volume. Then we should face the question whether the lattice size $(M=8,12)$ considered in [2] are sufficiently large to detect the IR renormalon factorial growth.

In order to have a simple estimation of the finite volume effect we consider the typical integral involving an IR renormalon and we introduce an infrared cutoff related to the finite volume. For a condensate with dimension $\delta$ we consider

$$
W_{\delta}\left(\alpha_{S}, M\right) \equiv \int_{k_{\mathrm{ir}}^{2}}^{k_{\mathrm{uv}}^{2}} \frac{d k^{2}}{k^{2}}\left(\frac{k}{k_{\mathrm{uv}}}\right)^{\delta} \alpha_{S}\left(k^{2}\right), \quad k_{\mathrm{uv}}=\frac{\pi}{a}, \quad k_{\mathrm{ir}}=\frac{2 \pi}{M a},
$$

with $k_{\mathrm{uv}}$ and $k_{\text {ir }}$ the infrared and ultraviolet cutoff on the lattice of size $M$ and spacing $a$. Using the one loop form of the running coupling

$$
\alpha_{S}\left(k^{2}\right)=\frac{\alpha_{S}}{1+b_{0} \alpha_{S} \ln \left(k^{2} / k_{\mathrm{uv}}^{2}\right)},
$$

one obtains the formal expansion

$$
W_{\delta}\left(\alpha_{S}, M\right)=\sum_{\ell=1} \alpha_{S}^{\ell} c_{\ell}(M), \quad c_{\ell}(M)=b_{0}^{\ell-1} \int_{k_{\mathrm{ir}}^{2}}^{k_{\mathrm{uv}}^{2}} \frac{d k^{2}}{k^{2}}\left(\frac{k}{k_{\mathrm{uv}}}\right)^{\delta} \ln ^{\ell-1}\left(\frac{k_{\mathrm{uv}}^{2}}{k^{2}}\right) .
$$

The factorial growth of the coefficients is obtained from values of $k$ around the steepest descent point

$$
k_{\mathrm{sd}}=k_{\mathrm{uv}} e^{-(\ell-1) / \delta} .
$$

\footnotetext{
$\dagger$ Since the stochastic method requires the introduction of two copies of the field for each order in $\alpha_{S}$, the amount of computer memory grows proportionally to $M^{4} \times$ number of loops. This is why only rather small lattices have been considered so far.

$\ddagger$ This problem has been stressed to us by Martin Beneke.
} 
The IR renormalon factorial growth is then obtained only if $k_{\mathrm{sd}}>k_{\mathrm{ir}}$, i.e. the steepest descent point lies inside the integration region, which gives the upper bound

$$
\ell<\ell_{\mathrm{sd}}=\delta \ln \left(\frac{M}{2}\right)+1
$$

Above this value the factorial growth is tamed. For $M=8,12$ and $\delta=4$ one obtains $\ell_{\mathrm{sd}} \simeq 6.5,8.2$. Therefore, among the coefficients computed in [2] only the ones with $\ell \leq 6$ would be inside the bound. For higher order coefficients one should use lattices larger than $M=8$.

In this paper we try to probe the reliability of the indication (41) and we shall argue that the factorial growth of the coefficients is still present at values of $\ell$ much larger than $\ell_{\text {sd }}$.

First we consider the exactly solvable asymptotically free case given by the large $N$ limit of the $O(N)$ non-linear sigma model in a two dimensional finite lattice. We consider the perturbative expansion of the condensate with $\delta=2$ and we compare the exact coefficients and their behaviour in $M$ with the ones given by (2). We show that the large order coefficients agree with the ones obtained from the typical integral (1) provided that the running coupling $\alpha_{S}\left(k^{2}\right)$ in the integrand is replaced by $\alpha_{S}\left(r k^{2}\right)$ with $r<1$. We then apply this idea to the four dimensional case and consider the expression in (11) with $\delta=4$. As suggested by the non-linear sigma model, we assume that the running coupling is taken at a scale smaller than $k^{2}$. We then argue that the factorial growth extends to larger values of $\ell$ well inside the values considered in [2].

\section{Non-linear $O(N)$ sigma model at large $N$}

IR renormalons are present in the perturbative expansion of any observable in the non-linear sigma model in two dimensions [6]. We consider the lattice version of the model [7] on a finite volume. The internal energy density $U$, which is a condensate of dimension $\delta=2$, has the following operator product expansion

$$
U=a^{-2} U_{\text {pert }}+m^{2} U_{1}+m^{4} U_{2}+\cdots,
$$

with $a$ the lattice UV cutoff and $m$ the scale parameter generated by the breaking of scale invariance. In the limit $N \rightarrow \infty$ one has

$$
(a m)^{2}=32 e^{-4 \pi \beta},
$$

where $\beta$ is the strong-coupling parameter at the lattice UV scale. The perturbative component has the following large $N$ expansion

$$
U_{\text {pert }}=\frac{N-2}{2 N} 4 \pi \alpha+\frac{1}{2 N} W(\alpha)+O\left(N^{-2}\right),
$$

where we have defined the weak-coupling by $4 \pi \alpha=1 / \beta$.

The first $1 / N$ correction contains IR renormalons. For finite volume, with $M$ lattice size, and for infinite volume one finds

$$
\begin{aligned}
& W(\alpha, M)=\frac{1}{M^{2}} \sum_{n_{1}, n_{2}} \frac{4 \pi \alpha}{1+4 \pi \alpha A_{M}\left(k_{1}, k_{2}\right)}, \\
& W(\alpha, \infty)=\int_{-\pi / a}^{\pi / a} \frac{a^{2} d^{2} k}{(2 \pi)^{2}} \frac{4 \pi \alpha}{1+4 \pi \alpha A_{\infty}\left(k_{1}, k_{2}\right)}
\end{aligned}
$$


with

$$
\begin{aligned}
& A_{M}\left(k_{1}, k_{2}\right)=\frac{1}{2 M^{2}} \sum_{m_{1}, m_{2}} \frac{\hat{k}^{2}-\hat{p}^{2}-(\widehat{p+k})^{2}}{\hat{p}^{2}(\widehat{p+k})^{2}}, \\
& A_{\infty}\left(k_{1}, k_{2}\right)=\frac{1}{2} \int_{-\pi / a}^{\pi / a} \frac{a^{2} d^{2} p}{(2 \pi)^{2}} \frac{\hat{k}^{2}-\hat{p}^{2}-(\widehat{p+k})^{2}}{\hat{p}^{2}(\widehat{p+k})^{2}} .
\end{aligned}
$$

As usual we have introduced the lattice momenta

$$
\hat{p}_{i}=\frac{2}{a} \sin \frac{a}{2} p_{i}, \quad \widehat{p+k_{i}}=\frac{2}{a} \sin \frac{a}{2}\left(p_{i}+k_{i}\right),
$$

with

$$
p_{i}=\frac{2 \pi n_{i}}{M a}, \quad k_{i}=\frac{2 \pi m_{i}}{M a}, \quad n_{i}, m_{i}=0, \pm 1, \pm 2, \cdots \pm(M / 2-1), M / 2 .
$$

for $M$ even and $\left|p_{i}\right|<\pi / a$ for $M \rightarrow \infty$. Notice that for finite volume the vanishing momenta do not contribute to the sums in (8) and (9) and then one has an IR cutoff

$$
p_{i}, k_{i} \leq k_{\mathrm{ir}}=\frac{2 \pi}{M a}
$$

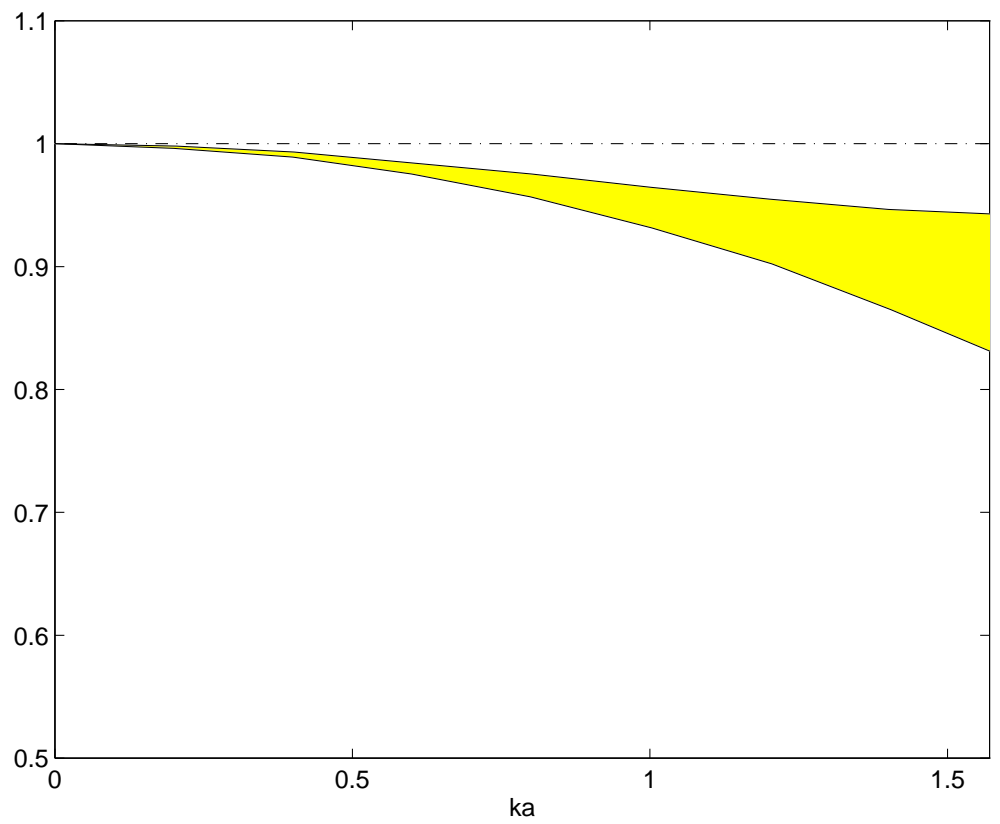

Figure 1: The ratio between $A_{256}\left(k_{1}, k_{2}\right)$ and its leading term of Eq. (11) plotted against $k a$; the spread for large $k^{2}$ is a lattice artifact representing the breaking of rotational invariance while the $O\left(k^{2}\right)$ term accounts for the curvature.

We now discuss the perturbative coefficients

$$
W(\alpha, M)=\sum_{\ell=1} \alpha^{\ell} C_{\ell}(M), \quad C_{\ell}(M)=\frac{4 \pi}{M^{2}} \sum_{n_{1}, n_{2}}\left(-4 \pi A_{M}\left(k_{1}, k_{2}\right)\right)^{\ell-1} .
$$

Consider first the infinite volume case. For small momenta $a\left|k_{i}\right| \ll 1$ one finds

$$
A_{\infty}\left(k_{1}, k_{2}\right) \simeq-\frac{1}{4 \pi} \ln \left(\frac{r k^{2}}{k_{\mathrm{uv}}^{2}}\right)+\mathcal{O}\left(a^{2} k^{2}\right), \quad r=\frac{\pi^{2}}{32},
$$


where $k^{2}=k_{1}^{2}+k_{2}^{2}$ (see fig. 1 ).

Therefore the integrand of (8) at small $k_{i}$ is

$$
\frac{4 \pi \alpha}{1+4 \pi \alpha A_{\infty}\left(k_{1}, k_{2}\right)} \simeq \frac{4 \pi \alpha}{1+b_{0} \alpha \ln \left(r k^{2} / k_{\mathrm{uv}}^{2}\right)}=4 \pi \alpha\left(r k^{2}\right)
$$

with $b_{0}=1$ in this case (see (6)). Therefore the integrand is given by the typical form (2) except that the running coupling is taken at the scale $r k^{2}$ smaller than $k^{2}$. The presence of the Landau pole is directly responsible for the factorial behaviour at large order

$$
C_{\ell}(\infty) \simeq b_{0}^{\ell-1}(\ell-1) ! r^{-1}, \quad \ell \gg 1 .
$$

Non-leading corrections in $1 / \ell$ can be estimated from (12) by taking into account that in the soft region the integrand $A_{\infty}\left(k_{1} k_{2}\right)$ approaches the running coupling at a scale $r k^{2}$ rather than $k^{2}$.

We consider now the exact evaluation of the perturbative coefficients $C_{\ell}(M)$ given by (10) both for $M$ finite and infinite.



Figure 2: The expansion coefficients $C_{\ell}$ for various lattice sizes $M$.

In fig. 2 we report the exact coefficients $C_{\ell}(M)$ as function of $\ell$ for various $M$ up to $M=256$; for relatively large loops $(\ell \gtrsim 4)$ and lattice size $(M \gtrsim 16)$ the exact coefficients $C_{\ell}(M)$ are fitted quite well by the expressions

$$
\bar{c}_{\ell}(M, r)=b_{0}^{\ell-1} \int_{k_{\mathrm{ir}}^{2}}^{k_{\mathrm{uv}}^{2}} \frac{d k^{2}}{k^{2}}\left(\frac{k}{k_{\mathrm{uv}}}\right)^{\delta} \ln \left(\frac{k_{\mathrm{uv}}^{2}}{r k^{2}}\right)^{\ell-1},
$$

with $\delta=2$ and $r$ given by the soft limit (11) of the integrand $r=\pi^{2} / 32$ (see fig. 3).

At finite $M$ the factorial behaviour of the coefficients is tamed by the IR cutoff $k_{\mathrm{ir}}$. To see this we plot in fig. 4 the ratio of the exact coefficients $R_{\ell}(M)=C_{\ell}(M) / C_{\ell}(\infty)$ as a function of $M$ for various $\ell$. As expected (see the discussion based on steepest descent estimation) this ratio vanishes by increasing $M$ at fixed $\ell$ or by increasing $\ell$ at fixed $M$. 


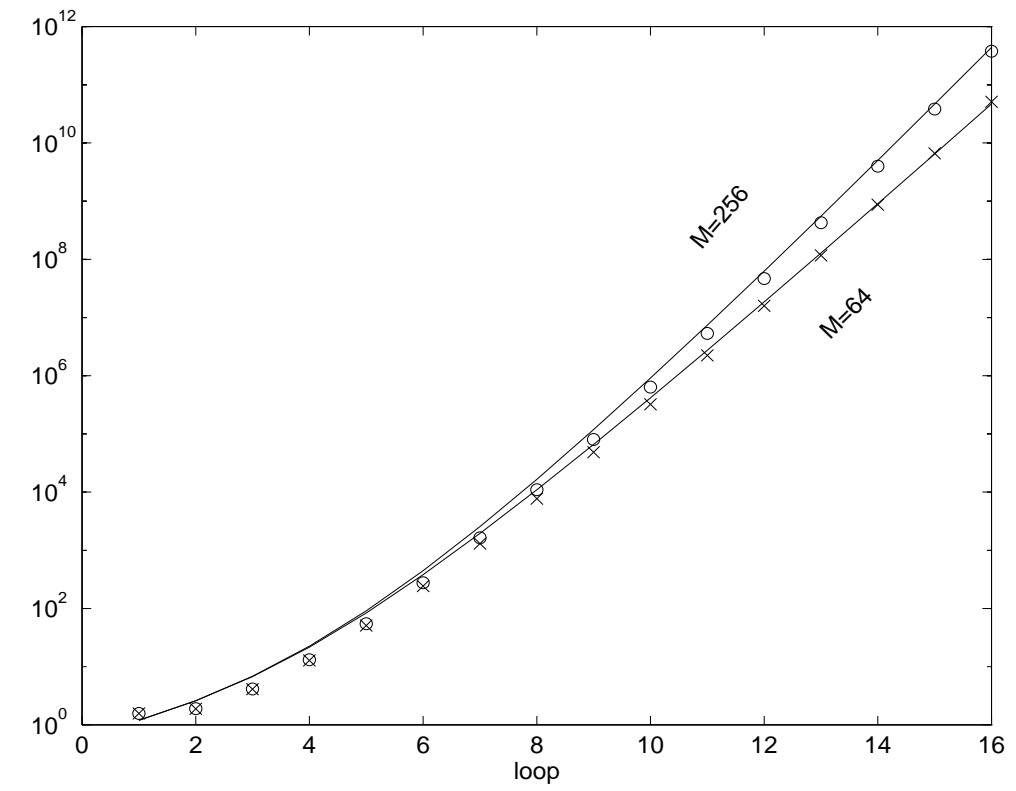

Figure 3: The exact coefficients $C_{\ell}$ and its approximate value given by Eq. (14) for $M=64(+)$ and $M=256(\mathrm{o})$.

We come now to analyze the question whether a reliable estimate of the IR renormalon behaviour can be obtained from finite lattice calculations. In particular we analyze the limitation (国) suggested by the steepest descent estimation.

In fig. 5 we plot $\ell_{\mathrm{sd}}$ as a function of $M$. At any given $M$ we report also the maximum value of $\ell$ such that the exact coefficient $C_{\ell}(M)$ is larger than a given fraction of the infinite volume exact coefficient $C_{\ell}(\infty)$. We plot the maximum $\ell$ at which $R_{\ell}(M)>\sigma$ with $\sigma=0.1,0.2$, and 0.5.

The finite volume clearly tames the growth of the exact coefficients at large $\ell$. However the factorial growth does not stop abruptly and is still present for $\ell$ larger than the limiting value $\ell_{\text {sd }}$ given in (舟). For instance, for the values of $M$ considered in fig. 5 , one finds that the exact finite volume coefficients $C_{\ell}(M)$ are larger than $20 \%$ of the infinite volume coefficient $C_{\ell}(\infty)$ for all values of $\ell$ with $\ell \lesssim \ell_{\text {sd }}+2$.

\section{Extension to the Yang-Mills case and conclusions}

We consider a naive extension of the previous analysis to the gluon condensate in the Yang-Mills theory on a four dimensional lattice. To this end, taking into account that the expression (14) fits very well the exact coefficients in the non-linear sigma model, we assume that also the gluon condensate coefficients are approximated by the expression (14) with $\delta=4$.

For $r$ we assume the same value as in the non-linear sigma model. In fig. 6 we plot the case of the gluon condensate. In the four dimensional case, as expected, the effect of the infrared cutoff is less severe and the factorial growth is still present for much larger values of $\ell$. For instance, in the four dimensional case the finite volume coefficients are larger than $20 \%$ of the infinite volume coefficients for all values of $\ell$ with $\ell \lesssim \ell_{\mathrm{sd}}+4$. 


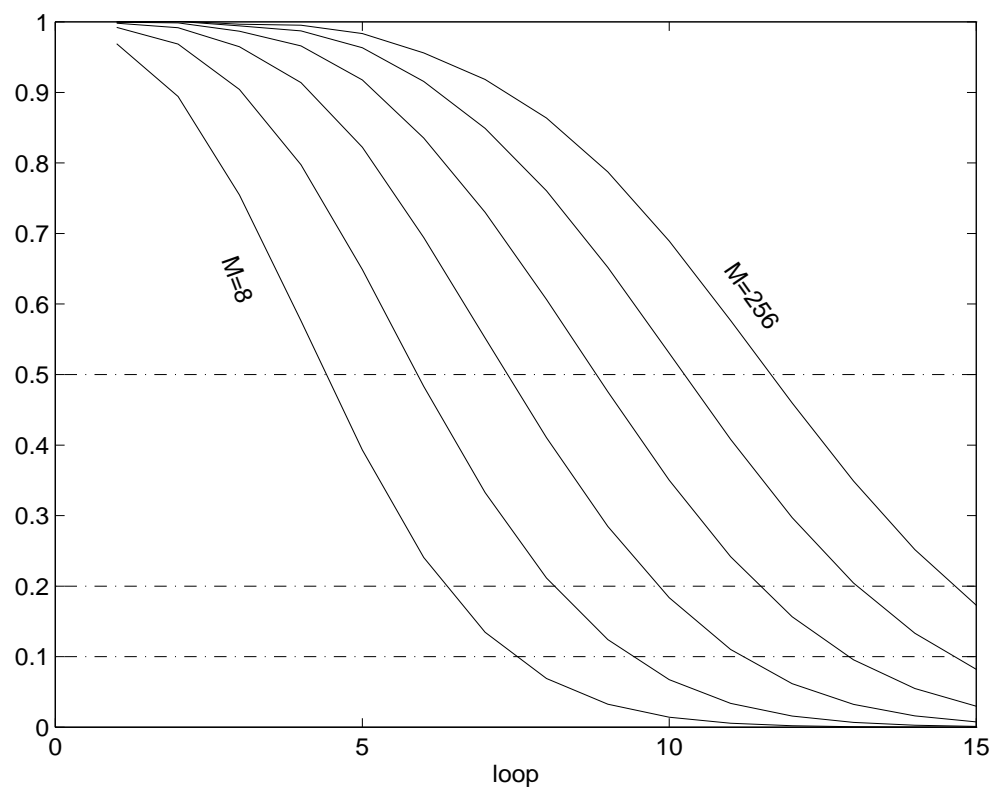

Figure 4: The ratios $C_{\ell}(M) / C_{\ell}(\infty)$.

We conclude that, if our fitted expression (14) for the gluon condensate perturbative coefficients is correct, the eight loop coefficient numerically computed in [2] is of the order of $50 \%$ of the infinite volume limit.

Acknowledgments

We are most grateful for valuable discussions with M. Beneke and G. Burgio.

\section{References}

[1] B. Allés, M. Campostrini, A. Feo and H. Panagopoulos, Phys. Lett. 324B (1994) 443 and references therein.

[2] F. Di Renzo, E. Onofri, and G. Marchesini, Nucl. Phys. B457 (1995) 202.

[3] B. Lautrup, Phys. Lett. 69B (1977) 109; G. Parisi, Phys. Lett. 76B (1977) 65; Nucl. Phys. B150 (1979) 163; G. t'Hooft, in The Whys of Subnuclear Physics, Erice 1977, ed A. Zichichi, (Plenum, New York 1977); V.I. Zakharov, Nucl. Phys. B385 (1992) 452; M. Beneke and V.I. Zakharov, Phys. Lett. 312B (1993) 340; M. Beneke Nucl. Phys. B307 (1993) 154; A. H. Mueller, Nucl. Phys. B250 (1985) 327; Phys. Lett. 308B (1993) 355; in QCD 20 years later, ed. P.M. Zerwas and H.A. Kastrup (World Scientific, Singapore 1993); G. Grunberg, Phys. Lett. 304B (1993) 183; Phys. Lett. 325B (1994) 441.

[4] G. Martinelli and C.T. Sachrajda, Nucl. Phys. B478 (1996) 660, C.T. Sachrajda, Nucl. Phys. B(PS) 47 (1996) 100.

[5] G. Parisi and Wu Yongshi, Sci. Sinica 24 (1981) 35. 


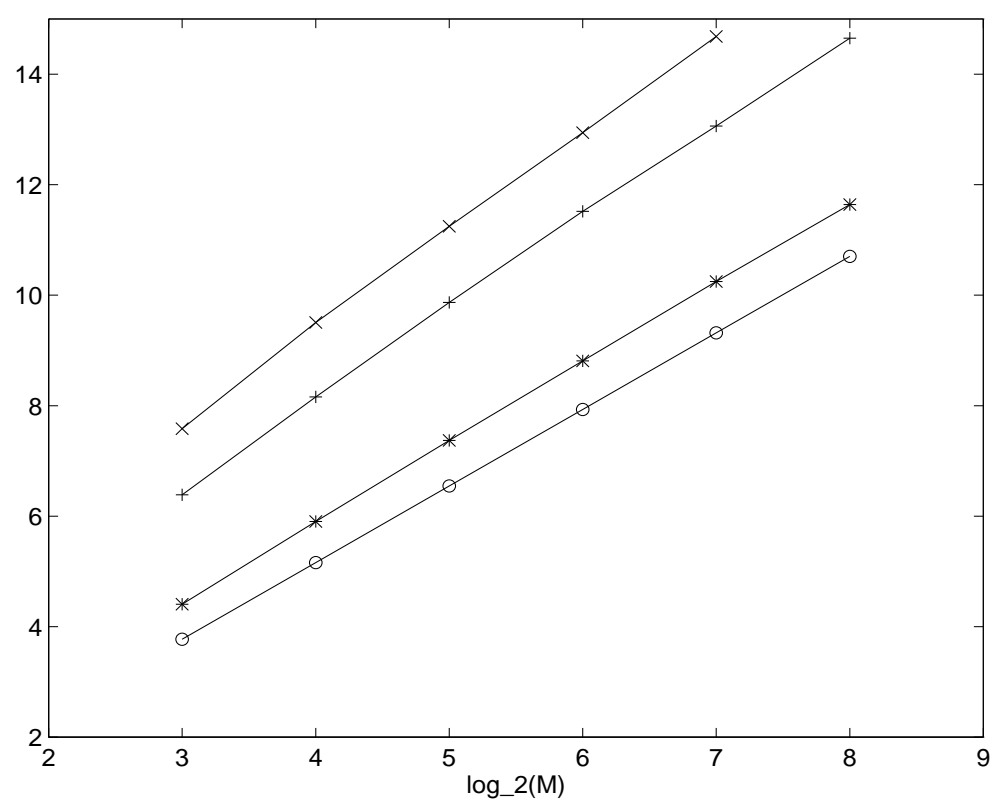

Figure 5: The loop order attainable with a prescribed fraction $\sigma=.1(\mathrm{x}), .2(+), .5(*)$ compared with the estimate $\ell_{s d}$.

[6] F. David, Nucl. Phys. B234 (1984) 237; Nucl. Phys. B263 (1986) 637;

V.A. Novikov, M.A. Shifman, A.I. Vainstein and V.I. Zakharov, Phys. Rep. 116 (1984) 105; Nucl. Phys. B249 (1985) 445.

[7] M. Campostrini and P. Rossi, Phys. Lett. 242B (1990) 81, Phys. Lett. 242B (1990) 225, Riv. Nuovo Cim. 16 (1993) 1. 


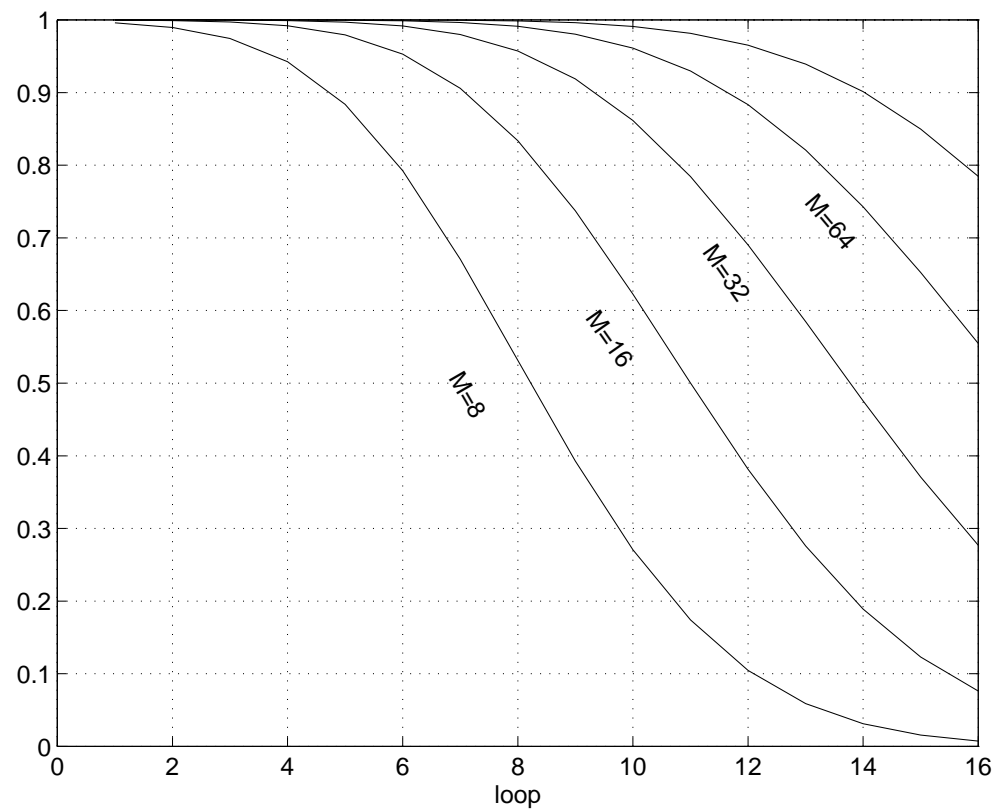

Figure 6: A possible scenario for $\delta=4$ of the ratios $\bar{c}_{\ell}(M) / \bar{c}_{\ell}(\infty)$ as obtained from Eq. (14) with $\delta=4$. 